\title{
On laminar groups, Tits alternatives and convergence group actions on $S^{2}$
}

\author{
Juan Alonso, Hyungryul Baik and Eric Samperton \\ Communicated by Pierre-Emmanuel Caprace
}

\begin{abstract}
Following previous work of the second author, we establish more properties of groups of circle homeomorphisms which admit invariant laminations. In this paper, we focus on a certain type of such groups, so-called pseudo-fibered groups, and show that many 3-manifold groups are examples of pseudo-fibered groups. We then prove that torsion-free pseudo-fibered groups satisfy a Tits alternative. We conclude by proving that a purely hyperbolic pseudo-fibered group acts on the 2-sphere as a convergence group. This leads to an interesting question if there are examples of pseudo-fibered groups other than 3-manifold groups.
\end{abstract}

\section{Introduction}

Thurston [25] showed that, if $M$ is an atoroidal 3-manifold admitting a taut foliation, then $\pi_{1}(M)$ acts faithfully on $S^{1}$ with a pair of dense invariant laminations. The result was generalized by Calegari-Dunfield [6], and one can find a complete treatment in [5]. Motivated by these results, the second author studied groups acting faithfully on $S^{1}$ with prescribed types and numbers of invariant laminations, in the process giving a new characterization of Fuchsian groups [1]. In the same paper, he asked if there exists a way of characterizing fibered 3-manifold groups in a similar way.

For a 3-manifold $M$ which fibers over the circle, one can construct a natural action of $\pi_{1}(M)$ on the circle with two invariant laminations, and this motivates the following definition. We call a subgroup $G$ of $\mathrm{Homeo}^{+}\left(S^{1}\right)$ pseudo-fibered if its action on $S^{1}$ admits two invariant, very full, loose laminations with distinct endpoints. As we said, this includes a large class of examples coming from 3manifolds which fiber over the circle, and the purpose of this paper is to show that pseudo-fibered groups in general have many nice properties.

We emphasize that, in this paper, all group actions on $S^{1}$ are considered up to conjugacy not semi-conjugacy (compare [20]). This is because it is not clear if the

The second author was partially supported by the ERC Grant No. 10160104 and Samsung Science \& Technology Foundation grant No. SSTF-BA1702-01. 
notion of pseudo-fibering is invariant under semi-conjugacy. See Section 2 for the precise definitions and relevant discussions.

In Section 4, we establish our first main result, a Tits alternative for torsion-free pseudo-fibered groups.

Theorem A. Let $G$ be a torsion-free pseudo-fibered group. Each subgroup of $G$ either contains a non-abelian free subgroup or is virtually abelian.

This is proved in Section 4.2 by studying how two elements of a (torsion-free) pseudo-fibered group interact with each other dynamically. In particular, we show a kind of dynamical alternative for elements of a pseudo-fibered group.

Theorem B. Let $G$ be a torsion-free pseudo-fibered group, and, for $f \in G$, let $\operatorname{Per}_{f}$ denote the set of all periodic points of $f$ on $S^{1}$. Then, for any $g, h \in G, \operatorname{Per}_{g}$ and $\mathrm{Per}_{h}$ are either equal or disjoint.

Theorem A will follow from Theorem B by applying the ping-pong lemma, and Hölder's theorem that a group acting faithfully and freely on $\mathbb{R}$ is necessarily abelian.

In Section 5, we study pseudo-fibered groups with more structure, inspired by (quasi-)Fuchsian groups. This part should be considered as part of Fenley's program which generalizes the work of Cannon-Thurston [8] considerably from the viewpoint of pseudo-Anosov flows (see [12]).

Our main result of Section 5 connects the pseudo-fibered group action on $S^{1}$ with a convergence group action on $S^{2}$. Note that a similar idea has been carried out in Fenley's program (see [12,14], and also compare [13, 15]).

Theorem C. Let $G$ be a pseudo-fibered group which is purely hyperbolic. Then $G$ acts on $S^{2}$ as a convergence group.

For the definition of "purely hyperbolic", see Section 2. We expect that Theorem $C$ can be strengthened. Indeed, when $G$ is a purely hyperbolic pseudo-fibered group, the second author has previously conjectured that $G$ is a Fuchsian group, hence acts on $S^{1}$ as a convergence group [1]. Recall that the work of many authors (e.g., $[9,16,26]$ ) shows that a group acts on $S^{1}$ as a convergence group if and only if it is topologically conjugate to a Fuchsian group.

Theorem A, Theorem B and Theorem C show that pseudo-fibered groups have properties similar to those of fibered 3-manifold groups. On the other hand, we provide a source of examples of pseudo-fibered groups which are quite different from fibered 3-manifold groups in Section 3. More precisely, in Theorem 3.1, we show that the free product of any two finite cyclic groups is a pseudo-fibered 
group. In the context of 3-manifolds, this implies that the fundamental group of the connected sum of any two lens spaces is also pseudo-fibered (Corollary 3.4).

Given the results above and the result of [1], we propose the following:

Conjecture 1.1 (Promotion of pseudo-fibering). Let $G$ be a finitely-generated torsion-free pseudo-fibered group which does not split as a non-trivial free product. Then there are three possibilities.

(1) $G$ is elementary, i.e., virtually abelian.

(2) $G$ is topologically conjugate to a Möbius group action (as usual, we consider $\mathrm{PSL}_{2}(\mathbb{R})$ a subgroup of $\mathrm{Homeo}^{+}\left(S^{1}\right)$ ).

(3) $G$ is abstractly isomorphic to a closed hyperbolic 3-manifold group.

A similar conjecture was made in [1], and the difference is discussed in Section 3. Here $G$ is said to be elementary if it is virtually abelian. This definition makes sense due to Theorem A, which asserts that every non-elementary pseudofibered group contains a non-abelian free subgroup.

\section{Preliminaries}

We briefly review and motivate several definitions regarding laminations on the circle. Two pairs $(a, b)$ and $(c, d)$ of distinct points of the circle $S^{1}$ are said to be linked if each connected component of $S^{1} \backslash\{a, b\}$ contains precisely one of $c, d$. They are called unlinked if they are not linked. Let $\mathcal{M}$ denote the set of all unordered pairs of two distinct points of $S^{1}$, i.e.,

$$
\mathcal{M}=\left(S^{1} \times S^{1}-\Delta\right) /(x, y) \sim(y, x),
$$

where $\Delta$ is the diagonal $\left\{(x, x): x \in S^{1}\right\}$. A lamination of $S^{1}$ is a closed subset of $\mathcal{M}$ whose elements are pairwise unlinked. Given a lamination $\Lambda$, an element $(a, b)$ of $\Lambda$ is called a leaf, and the points $a, b$ are called the endpoints of the leaf $(a, b)$ (or just endpoints of $\Lambda$ if there is no possible confusion). Two laminations have distinct endpoints if their sets of endpoints are disjoint. A lamination $\Lambda$ is called dense if the set of endpoints of $\Lambda$ is a dense subset of $S^{1}$.

Any subgroup $G$ of $\mathrm{Homeo}^{+}\left(S^{1}\right)$ has an induced action on $\mathcal{M}$. We say that a lamination $\Lambda$ is $G$-invariant if the $G$-action on $\mathcal{M}$ preserves $\Lambda$ set-wise. A discrete subgroup $G$ of $\mathrm{Homeo}^{+}\left(S^{1}\right)$ is called laminar if it admits a dense $G$-invariant lamination.

Let $\mathbb{D}$ denote the closed unit disk in $\mathbb{C}$, where the interior is equipped with the Poincaré metric, i.e., $\mathbb{D}=\mathbb{H}^{2} \cup \partial_{\infty} \mathbb{H}^{2}$. A lamination $\Lambda^{\prime}$ of $\mathbb{D}$ is a set of chords with disjoint interiors such that there exists a lamination $\Lambda$ in $S^{1}=\partial \mathbb{D}$, where the chords in $\Lambda^{\prime}$ can be obtained by connecting the endpoints of the leaves of $\Lambda$. 
As noted in [5, Construction 2.4], the set of laminations on $S^{1}$ and the set of geodesic laminations of $\mathbb{H}^{2}$ are in one-to-one correspondence up to isotopy relative to $S^{1}=\partial_{\infty} \mathbb{H}^{2}$. Hence we freely switch our viewpoint between these two without further mentioning. A gap of a lamination $\Lambda$ is the closure of a connected component of $\mathbb{H}^{2} \backslash \Lambda$ in $\mathbb{H}^{2} \cup \partial_{\infty} \mathbb{H}$.

We recall some key properties of laminations from [1] (also compare [4]) .

Definition 2.1. A lamination $\Lambda$ is said to be

- totally disconnected if no open subset of the disk is foliated by $\Lambda$,

- very full if each gap is a finite-sided ideal polygon in the disk,

- loose if no two leaves share an endpoint unless they are edges of the same (necessarily unique) gap.

For every element $f$ of $\operatorname{Homeo}^{+}\left(S^{1}\right)$, let $\mathrm{Fix}_{f} \subset S^{1}$ denote the set of all fixed points of $f$. Let $\operatorname{Per}_{f}$ denote the set of all periodic points of $f$, where a point $p$ of $S^{1}$ is periodic for $f$ if the orbit of $p$ under $f$ is finite. Thus $\operatorname{Fix}_{f} \subset \operatorname{Per}_{f}$. A fixed point $p$ of the homeomorphism $f$ is attracting if there exists an interval $I \ni p$ containing no other fixed points such that $f(I) \subsetneq I$. Similarly, a fixed point $q$ is repelling if there exists an interval $J \ni q$ containing no other fixed points such that $f(J) \supsetneq J$.

We first give names to particular types of homeomorphisms of $S^{1}$ in the following definition as in [1]. For the first four types of homeomorphisms, compare [19], where Möbius-like elliptic, Möbius-like parabolic, Möbius-like hyperbolic and Möbius-like homeomorphisms are defined.

Definition 2.2. An element $f$ of $\mathrm{Homeo}^{+}\left(S^{1}\right)$ is said to be

- elliptic if $f$ has no fixed points,

- parabolic if $f$ has a unique fixed point,

- hyperbolic if $f$ has two fixed points, one attracting and one repelling,

- Möbius-like if $f$ is conjugate in Homeo ${ }^{+}\left(S^{1}\right)$ to an element of $\operatorname{PSL}_{2}(\mathbb{R})$,

- pseudo-Anosov-like or $p$-A-like if $f$ is not hyperbolic and some positive power $f^{n}$ has a positive, even number of fixed points alternating between attracting and repelling,

- properly pseudo-Anosov-like or properly p-A-like if $f$ is pseudo-Anosov-like and non-elliptic.

Thus $f$ is $\mathrm{p}$-A-like if and only if a positive power of $f$ is properly p-A-like. For a p-A-like homeomorphism $f \in \mathrm{Homeo}^{+}\left(S^{1}\right)$, the set of boundary leaves of the 
convex hull of the attracting fixed points of a properly p-A-like power $f^{n}$ is called the attracting polygon of $f$. Similarly, $f$ has a repelling polygon.

Definition 2.3. Let $\Lambda$ be a lamination. A leaf $l \in \Lambda$ is said to be visible from a point $p \in S^{1}$ if one can connect $l$ to $p$ by a geodesic of $\mathbb{H}^{2}$ (i.e., there exists a geodesic ray from a point on $l$ to $p$ ) which does not intersect any leaf of $\Lambda$ in $\mathbb{H}^{2}$.

Observe that, if $p$ is an endpoint of a gap in a very full, loose lamination, the set of leaves visible from $p$ is precisely the set of edges of the gap.

Now we outline some examples of laminar groups mentioned in the introduction. Let $S$ be a closed hyperbolic surface, and let $\phi$ be a pseudo-Anosov homeomorphism of $S$. Let $M$ be the mapping torus $S \times[0,1] /(x, 1) \sim(\phi(x), 0)$. Then one can construct a faithful action of $\pi_{1}(M)$ on $S^{1}$ in the following way. Note $\pi_{1}(M)$ is isomorphic to $\pi_{1}(S) \rtimes \mathbb{Z}$. The deck transformation action of $\pi_{1}(S)$ on $\mathbb{H}^{2}$ extends continuously to a faithful action on $\partial \mathbb{H}^{2}$. Let $\widetilde{\phi}: \mathbb{H}^{2} \rightarrow \mathbb{H}^{2}$ be a lift of $\phi$ to the universal cover of $S$. Since $S$ has finite area, $\widetilde{\phi}$ is a quasi-isometry, hence extends to a homeomorphism on $\partial \mathbb{H}^{2}$. Considering this homeomorphism on $\partial \mathbb{H}^{2}$ as a generator of $\mathbb{Z}$, this defines an action

$$
\rho: \pi_{1}(M)=\pi_{1}(S) \rtimes \mathbb{Z} \rightarrow \text { Homeo }^{+}\left(\partial \mathbb{H}^{2}\right)=\text { Homeo }^{+}\left(S^{1}\right) .
$$

Then $\rho\left(\pi_{1}(M)\right)$ is laminar since it fixes both the stable and unstable laminations of $\phi$. In fact, $\rho$ is faithful.

Definition 2.4. A finitely generated laminar group $G$ is said to be fibered if $G$ is topologically conjugate to $\rho\left(\pi_{1}(M)\right)$ where $\rho$ and $M$ are as in the previous paragraph, and the conjugacy takes the $G$-invariant lamination to one of the invariant laminations of the monodromy of $M$.

Definition 2.5. A finitely generated laminar group $G$ is said to be pseudo-fibered if it preserves a pair of very full loose invariant laminations $\Lambda_{1}, \Lambda_{2}$ with distinct endpoints, and each non-trivial element of $G$ has at most countably many fixed points in $S^{1}$. We also say $\left(G, \Lambda_{1}, \Lambda_{2}\right)$ is a pseudo-fibered triple.

Pseudo-fibered groups were first studied in [1]; although they had not yet been given a name.

Theorem 2.6 ([1, Section 8]). Let $\left(G, \Lambda_{1}, \Lambda_{2}\right)$ be a pseudo-fibered triple. Let $g \in G$. Then

(1) $g$ is either Möbius-like or pseudo-Anosov-like,

(2) if $g$ is $p$-A-like, then, for some $i, j=1,2$ with $i \neq j, \Lambda_{i}$ contains the attracting polygon of $g$ and $\Lambda_{j}$ contains the repelling polygon of $g$. 
Furthermore, [1] shows that, if $G$ is torsion-free, then all Möbius-like elements are hyperbolic elements.

The following proposition justifies the term "pseudo-fibered".

\section{Proposition 2.7. A fibered group $G$ is pseudo-fibered.}

Proof. We only need to worry about the cardinality of the set of fixed points of each element. But this is not a problem due to [10, Theorem 5.5] which asserts that, for a given pseudo-Anosov surface homeomorphism $h$, any lift of a strictly positive power of $h$ has finitely many fixed points on $\partial_{\infty} \mathbb{H}^{2}$, alternating between attracting and repelling.

In fact, the proof of [10, Theorem 5.5, pp. 85-87] shows Theorem 2.6 in the case of fibered groups. Any lift of a strictly positive power of $h$ falls into one of the three cases. Case 1 and Case 2 correspond to properly pseudo-Anosov-like elements, and Case 3 corresponds to hyperbolic elements in the sense of Definition 2.2. In Case 1, the attracting repelling polygons of the p-A-like element have three or more sides, and, in Case 2, those polygons are degenerate, i.e., there are exactly two attracting fixed points and two repelling fixed points.

We remark that the "pseudo" in "pseudo-fibered group" intentionally carries two different connotations. The first, as in Theorem 2.6, indicates that some elements are pseudo-Anosov-like. The second, as in Conjecture 1.1, indicates that pseudo-fibered groups are (conjecturally) not far from fibered groups.

In Section 5, we study a special class of pseudo-fibered groups.

Definition 2.8. Let $G$ be a pseudo-fibered group. Then $G$ is called purely hyperbolic if it has no pseudo-Anosov-like elements.

Theorem $\mathrm{C}$ says that a purely hyperbolic pseudo-fibered group acts on the sphere as a convergence group. In general, a group $G$ acting on a compactum $X$ is called a discrete convergence group if the following holds: for any infinite sequence of distinct elements $\left(g_{i}\right)$ of $G$, there exists a subsequence $\left(g_{i_{j}}\right)$ of $\left(g_{i}\right)$ and two points $a, b \in X$ not necessarily distinct such that $g_{i_{j}}$ converges to the constant map with value $a$ uniformly on every compact subset of $X \backslash\{b\}$, and $g_{i_{j}}^{-1}$ converges to the constant map with value $b$ uniformly on every compact subset of $X \backslash\{a\}$. Since we only deal with discrete convergence groups in this paper, we will omit the word discrete and simply call it a convergence group.

As mentioned before, when $X$ is $S^{1}$, being a convergence group is equivalent to being (conjugate to) a Fuchsian group. This result is known as the convergence group theorem $[9,16,26]$, and the same statement for indiscrete convergence groups was proved in [18]. 
Remark 2.9. There is a well-known equivalent definition of a convergence group action. A group $G$ acting on $X$ is called a convergence group if the diagonal action of $G$ on $X \times X \times X \backslash \Delta$ is properly discontinuous, where $\Delta$ is the set of triples of points of $X$ which are not all distinct. See, for instance, [27]. If the diagonal action on the set of distinct triples is also cocompact, then $G$ is called a uniform convergence group.

Remark 2.10. When $X$ is $S^{1}$, it is easy to see that the uniform convergence in the definition of a convergence group can be replaced by the pointwise convergence. However, it is important not to conflate the notions of "uniform convergence group" and "uniform convergence".

Remark 2.11. When $X$ is $S^{2}$, the analogue of the convergence group theorem is not true, i.e., not every discrete convergence group action on $S^{2}$ comes from a Kleinian group. For instance, one can just start with a Fuchsian representation of a surface group into $\mathrm{PSL}_{2}(\mathbb{C})$ and quotient the lower hemisphere to a single point. On the other hand, it is a famous open problem whether or not all uniform convergence group actions on $S^{2}$ come from Kleinian groups.

Finally, the idea of a rainbow, first described in [1], will be useful throughout this paper.

Definition 2.12. A lamination $\Lambda$ is said to have a rainbow at a point $p \in S^{1}$ if there is a sequence of leaves $\left(l_{i}\right)=\left(\left(a_{i}, b_{i}\right)\right)$ of $\Lambda$ such that $\left(a_{i}\right)$ and $\left(b_{i}\right)$ converge to $p$ from opposite sides. Such a sequence $\left(l_{i}\right)$ is called a rainbow at $p$ in $\Lambda$.

A rainbow is a particularly nice way of approximating a point $p \in S^{1}$ by leaves of a lamination. Clearly, endpoints of leaves do not admit rainbows. On the other hand, an observation we shall use later is that, for a very full lamination $\Lambda$, these approximations exist for every point that is not an endpoint of a leaf.

Lemma 2.13 (Rainbow lemma [1, Theorem 5.3]). Let $\Lambda$ be a very full lamination of $S^{1}$. Every point $p \in S^{1}$ is either an endpoint of a leaf of $\Lambda$, or there is a rainbow in $\Lambda$ at $p$. These two possibilities are mutually exclusive.

\subsection{Semi-conjugacy destroys pseudo-fiberedness}

We remark in this subsection that semi-conjugacy appears to be irrelevant to the study of pseudo-fibered groups. This is to be expected in the context of our promotion of the pseudo-fibering conjecture since semi-conjugacy does not preserve convergence actions. In particular, our Theorem A should be seen as distinct from 
Margulis's Tits alternative for minimal subgroups of $\mathrm{Homeo}^{+}\left(S^{1}\right)$, a point we explain now.

A continuous surjective map $f: S^{1} \rightarrow S^{1}$ is said to be monotone if the preimage of each point is connected. For a group $G$, two actions

$$
\rho \quad \text { and } \quad \mu: G \rightarrow \text { Homeo }^{+}\left(S^{1}\right)
$$

are said to be semi-conjugate (or $\rho$ is semi-conjugate to $\mu$ ) if there exists a monotone map $f$ such that $f \circ \rho=\mu \circ f$. For many aspects of the theory of groups of circle homeomorphisms, it is enough to consider the actions up to semi-conjugacy.

A classical theorem of Poincaré says that every subgroup of $\mathrm{Homeo}^{+}\left(S^{1}\right)$ either has a finite orbit or is semi-conjugate to a minimal action (meaning every orbit is dense). Furthermore, a theorem of Margulis says that subgroups of Homeo $^{+}\left(S^{1}\right)$ that act minimally either contain $F_{2}$ as a subgroup or are abelian [21]. (For more details regarding both of these results, as well as a general introduction to group actions on $S^{1}$, see [17].)

What we have shown is that, for a pseudo-fibered group, even if either there exists a finite orbit or the action is non-minimal, the Tits alternative always holds. Hence the scope of Theorem A is distinct from the Tits alternative of Margulis.

However, it is still an interesting question to ask if the pseudo-fibered groups can be studied up to semi-conjugacy. We observe that semi-conjugacy may destroy a pseudo-fibered triple since the laminations do not behave well under semiconjugacy. More precisely, we show the following:

Proposition 2.14. Let $f: S^{1} \rightarrow S^{1}$ be a monotone map which is not a homeomorphism, and let $\left(\Lambda_{1}, \Lambda_{2}\right)$ be a pair of laminations. At most one of the pairs, $\left(\Lambda_{1}, \Lambda_{2}\right)$ and $\left(f\left(\Lambda_{1}\right), f\left(\Lambda_{2}\right)\right)$, can be a pair of very full loose laminations with disjoint endpoint sets.

Proof. Since $f$ is not injective, there is a point $p \in S^{1}$ such that $I:=f^{-1}(p)$ has non-empty interior. Let $\hat{p}$ be an endpoint of $I$. Recall that the rainbow lemma says that, for each $p \in S^{1}$ and a very full lamination $\Lambda$, either $p$ is an endpoint of a leaf or there is a rainbow at $p$ in $\Lambda$.

Suppose $\left(\Lambda_{1}, \Lambda_{2}\right)$ is a pair of very full loose laminations with disjoint endpoint sets. In particular, there must be a rainbow at $\hat{p}$ in $\Lambda_{i}$ for at least one of the $i=1$ or 2. But the image of a rainbow at $\hat{p}$ under $f$ is an infinite set of leaves of $f\left(\Lambda_{i}\right)$ which share a common endpoint. Hence $f\left(\Lambda_{i}\right)$ cannot be loose.

For the other direction, suppose $\left(f\left(\Lambda_{1}\right), f\left(\Lambda_{2}\right)\right)$ is a pair of very full loose laminations with disjoint endpoint sets. From the above argument, we know that there is no rainbow at $\hat{p}$ in $\Lambda_{i}$ for each $i$. But this means $\hat{p}$ is an endpoint of some leaf in both $\Lambda_{1}$ and $\Lambda_{2}$; hence they cannot have disjoint endpoint sets. 


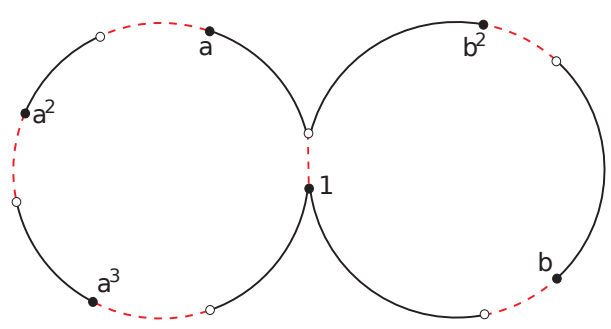

Figure 1. The seed graph $\Gamma_{0}$.

There are examples of pseudo-fibered triples whose actions are not minimal. Indeed, it is easy to construct examples of pseudo-fibered groups with finite orbits, and, in the next section, we construct examples of pseudo-fibered groups whose actions are neither minimal nor have finite orbits. It would be interesting to more thoroughly unravel the relationship between (non-)minimal actions, finite orbits and Conjecture 1.1. From this perspective, it is natural to ask the following:

Question 2.15. Is a pseudo-fibering semi-conjugacy invariant? Namely, for two semi-conjugate actions $\rho_{1}, \rho_{2}$ of a group $G$ on $S^{1}$, if $\rho_{1}$ is pseudo-fibered with two laminations $\Lambda_{1}, \Lambda_{2}$, is $\rho_{2}$ also pseudo-fibered with respect to a different pair of laminations $\Gamma_{1}, \Gamma_{2}$ ?

Note that, even if the above question has an affirmative answer, $\left(\Lambda_{1}, \Lambda_{2}\right)$ and $\left(\Gamma_{1}, \Gamma_{2}\right)$ may not be related in any obvious way as we saw in Proposition 2.14.

\section{Free products, torsion and promotion of pseudo-fibering}

Theorems A and $\mathrm{C}$ can be seen also as partial evidence for Conjecture 1.1. In [1], a conjecture similar to Conjecture 1.1 was made without free indecomposability assumption. The following theorem shows why Conjecture 1.1 was amended.

Theorem 3.1. Let $G, H$ be any finite cyclic groups. Then $G * H$ embeds into Homeo $^{+}\left(S^{1}\right)$ as a pseudo-fibered group.

Proof. This construction is adapted from a construction in [2] that yields a faithful action of any free product of subgroups of Homeo ${ }^{+}\left(S^{1}\right)$ on a new circle, which blows down onto each of the original circles. We content ourselves with a brief review of the ideas of [2] and a description of how to additionally construct invariant laminations.

To construct an action of $G * H$ on $S^{1}$, begin by forming two pointed copies of $S^{1}$ called $S_{G}^{1}$ and $S_{H}^{1}$, with marked points both denoted 1 . Let $G$ act on $S_{G}^{1}$ as 


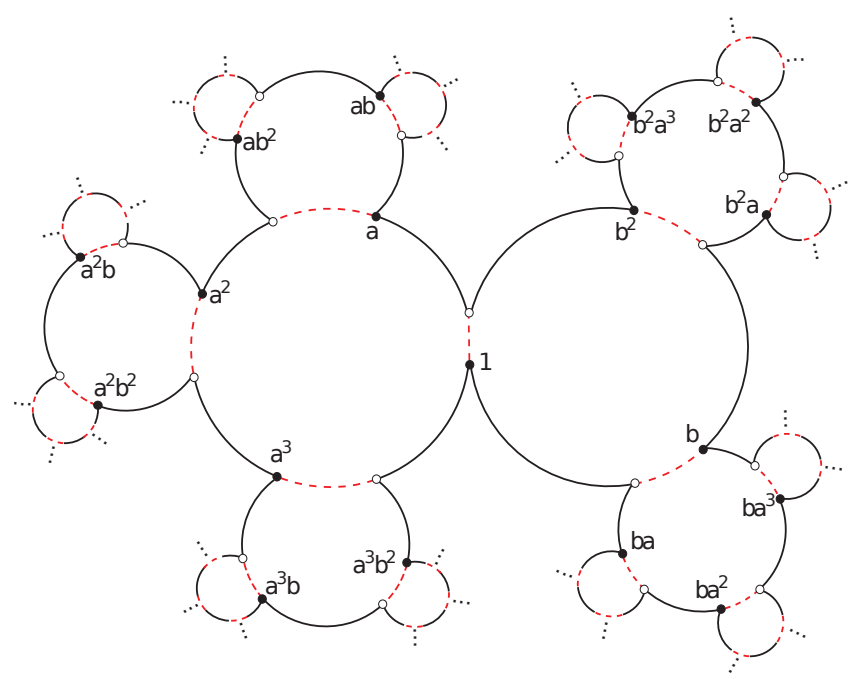

Figure 2 . The limiting circle $S^{1}=\overline{\Gamma_{\infty}}$ laminated by $\Lambda_{0}$, which is represented by the dashed red lines.

a finite rotation subgroup and $H$ act on $S_{H}^{1}$ as a finite rotation subgroup. The $G$ orbit of the point marked 1 in $S_{G}^{1}$ is now a copy of $G$, and the $H$-orbit of 1 in $S_{H}^{1}$ is a copy of $H$. Mark all of these points accordingly, wedge $S_{G}^{1}$ and $S_{H}^{1}$ together at the points marked by 1 , blow up all of the marked points, and consistently label one of the endpoints of the blow-up intervals. The resulting "seed", called $\Gamma_{0}$, is in Figure 1, where we have $G=\left\langle a \mid a^{4}=1\right\rangle$ and $H=\left\langle b \mid b^{3}=1\right\rangle$. Now generate an infinite graph $\Gamma_{\infty}^{\prime}$ on which $G * H$ acts faithfully. As in Figure 2, write $\Gamma_{\infty}^{\prime}=\Lambda_{0} \cup \Gamma_{\infty}$, where $\Lambda_{0}$ is the orbit of the blown-up intervals in $\Gamma_{0}$, and $\Gamma_{\infty}$ is everything else. The order completion $\overline{\Gamma_{\infty}}$ is $S^{1}$, and $\Lambda_{0}$ is a discrete lamination on this circle. This proves the following lemma:

Lemma 3.2. Let $G, H$ be any finite cyclic groups. Then there exists an injective homomorphism $\rho: G * H \rightarrow \operatorname{Homeo}^{+}\left(S^{1}\right)$ such that $\rho(G * H)$ admits a discrete invariant lamination.

Now one can easily add more leaves to $\Lambda_{0}$ to construct a $G * H$-invariant very full and loose lamination $\Lambda_{1}$. For example, in the left circle of the seed $\Gamma_{0}$, first take a polygon which has one vertex in each connected component of the complement of the dotted segments and is invariant under the action of $G$. Then, in the region between this polygon and an element of $\Lambda_{0}$ in $\Gamma_{0}$, add infinitely many triangles to make the lamination very full and loose in that region. Now fill the other such regions so that lamination becomes $G$-invariant. One can do the same thing 


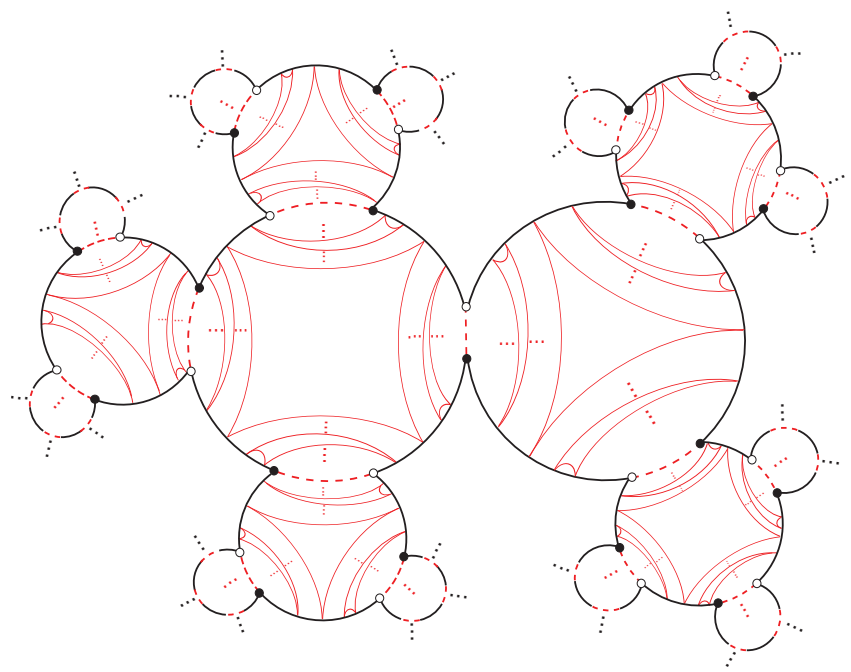

Figure 3. The very full and loose lamination $\Lambda_{1} \supset \Lambda_{0}$. We have removed the markings to avoid clutter.

for the right circle to get an $H$-invariant very full loose lamination and then extend it as a $G * H$-invariant lamination $\Lambda_{1}$ which contains $\Lambda_{0}$ as a sublamination. The final result is in Figure 3. Obviously, $\Lambda_{1}$ is very full and loose away from $\Lambda_{0}$. It is loose at $\Lambda_{0}$ because the leaves of $\Lambda_{0}$ are not contained in gaps - they are instead limits of gaps.

We need to construct another $G * H$-invariant very full loose lamination $\Lambda_{2}$ so that $\Lambda_{1}$ and $\Lambda_{2}$ have distinct endpoints. To build $\Lambda_{2}$, we first replace each leaf of $\Lambda_{0}$ with endpoints by four leaves forming, say, a rectangle such that each endpoint of the original dotted segment lies between two adjacent vertices of the rectangle. In the two regions between the rectangle and the endpoints of the original leaf in $\Lambda_{0}$, put infinitely many triangles to make the lamination very full and loose. These choices can obviously be made so that the endpoints of the new leaves are disjoint from $\Lambda_{1}$, and, by working in one region at a time, we can do the construction $G * H$-invariantly, resulting in Figure 4. In the regions where all the rectangles are visible, we do the exactly same thing as when constructing $\Gamma_{1}$ from $\Gamma_{0}$ : take a big invariant polygon, and fill out all the complementary regions. The result is shown in Figure 5.

Finally, to show that $\left(\rho(G * H), \Lambda_{1}, \Lambda_{2}\right)$ is a pseudo-fibered triple, we need to show that every element of $G * H=\rho(G * H)$ has countably many fixed points in its action on $S^{1}$. There are two ways to prove this, either using Bass-Serre theory or the existence of even more $G * H$-invariant laminations under this action. 


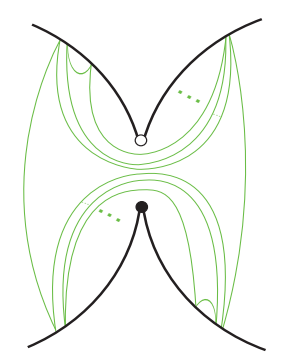

Figure 4. Replacing $\Lambda_{0}$ with rectangles and triangles.

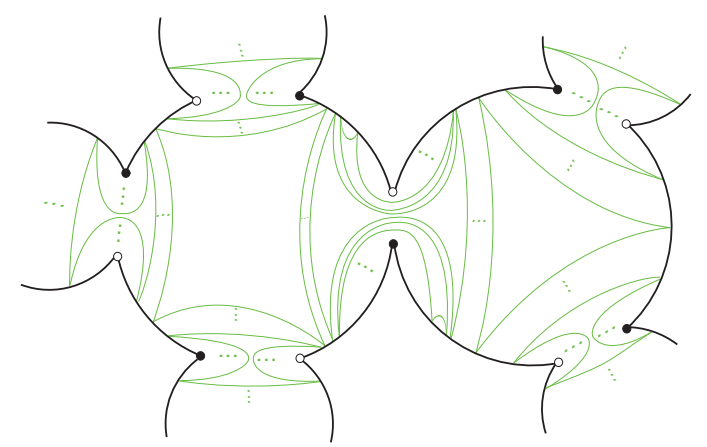

Figure 5. The very full and loose lamination $\Lambda_{2}$.

For the latter approach, we quote the following result.

Theorem 3.3 ([1]). Every subgroup of $\mathrm{Homeo}^{+}\left(S^{1}\right)$ admitting three very full invariant laminations with distinct endpoint sets is Möbius-like, meaning every element is (individually) Möbius-like.

Since both $G$ and $H$ are finite, there is a large freedom to construct laminations inductively as before. Indeed, we can slightly perturb the construction of $\Lambda_{2}$ to get a third very full and loose invariant lamination $\Lambda_{3}$ with endpoints distinct from $\Lambda_{1}$ and $\Lambda_{2}$. Theorem 3.3 then implies that every element of $G * H$ is Möbius-like, hence has finitely many fixed points.

Alternatively, to show that every element of $G * H$ acts on $S^{1}$ with at most two fixed points, we can use Bass-Serre theory. Clearly, torsion elements of $G * H$ act freely on $S^{1}$. Non-torsion elements must have their fixed points in the subset $S^{1} \backslash \Gamma_{\infty}$, which can be identified with the ends of the Bass-Serre tree for $G * H$. Standard results now imply that such elements have two fixed points in $S^{1}$.

Theorem 3.1 has an immediate corollary in the context of 3-manifold groups. 
Corollary 3.4. Let $M$ be a connected sum of two lens spaces. Then $\pi_{1}(M)$ admits a pseudo-fibered group action on $S^{1}$.

\section{The Tits alternative for pseudo-fibered groups}

Throughout this section, $G$ will denote a torsion-free pseudo-fibered group, except where explicitly indicated elsewhere. This will allow us to apply Theorem 2.6, which we may do sometimes without mentioning.

\subsection{Proof of Theorem B}

To prove that pseudo-fibered groups satisfy the Tits alternative, we first prove Theorem B, which, we recall, says that two elements of a pseudo-fibered group have either equal or disjoint sets of periodic points. This can be done, for instance, by analyzing how each element of the group acts on the quotient of the circle obtained by collapsing leaves of an invariant lamination. One can show that such a quotient is a dendrite as in [24], and this point of view has its own advantages. But, for our purpose, it is simpler to analyze the group action on the circle directly.

We will need a number of lemmas. The first follows immediately from the definitions, so we leave its proof to the reader.

Lemma 4.1. Suppose there are two dense laminations with distinct endpoints. Then each of the laminations is totally disconnected.

Recall that, by definition, a lamination $\Lambda$ is a type of closed subset of

$$
\left(S^{1} \times S^{1}-\Delta\right) /(x, y) \sim(y, x) .
$$

Thus it makes sense to talk about the neighborhood in $\Lambda$ of a leaf, isolated leaves, etc.

Lemma 4.2. Each leaf of a totally disconnected very full lamination is either a boundary leaf of a gap or is the limit of an infinite sequence of gaps.

Proof. This is a direct consequence of the definition of a very full lamination. Indeed, if a neighborhood of a leaf meets no gaps, it must be foliated, contradicting that the lamination is totally disconnected.

Lemma 4.3. Let $\Lambda$ be a totally disconnected very full lamination. Then $\Lambda$ is loose if and only if the following conditions are satisfied:

(i) For each $p \in S^{1}$, at most finitely many leaves of $\Lambda$ have $p$ as an endpoint.

(ii) There are no isolated leaves. 
Proof. Suppose $\Lambda$ is loose. Then each $p \in S^{1}$ is an endpoint of at most two leaves (maybe none) of $\Lambda$. Hence condition (1) follows immediately.

For condition (2), suppose there exists a leaf $L$ which is isolated. Let $J_{1}$ and $J_{2}$ be the connected components of the complements of the endpoints of $L$ in $S^{1}$. The fact that $L$ is isolated means there exists an open arc $I_{1}$ containing one endpoint of $L$, and another open $\operatorname{arc} I_{2}$ containing the other endpoint of $L$, such that, for each $i=1,2$, there exists no leaf connecting $I_{1} \cap J_{i}$ to $I_{2} \cap J_{i}$. For $i=1,2$, define $\Lambda_{i}$ to be the set of leaves of $\Lambda$ with endpoints in $J_{i}$ that are visible from both endpoints of $L$. Both $\Lambda_{i}$ are nonempty since $\Lambda$ is dense. For each $i=1,2$, $\Lambda_{i} \cup\{L\}$ is the set of boundary leaves of a gap $P_{i}$ of $\Lambda$. This contradicts looseness of $\Lambda$ since both $P_{1}$ and $P_{2}$ are gaps sharing some of their vertices.

Now, for the converse, assume $\Lambda$ satisfies conditions (1) and (2). Suppose $p$ is a common endpoint of two gaps. Let $L_{1}, L_{2}$ be the innermost leaves ending at $p$. (It is possible $L_{1}=L_{2}$, but this does not change what follows.) Then $L_{1}$ is either isolated (absurd by condition (2)), or is approximated by infinitely many leaves. Since these leaves cannot cross $L_{2}$, they must end at $p$, contradicting condition (1).

Lemma 4.4. Let $G$ be a pseudo-fibered group, and let $\Lambda$ be a very full loose $G$-invariant lamination. If $g$ is a hyperbolic element, then the fixed points of $g$ in $S^{1}$ are not an endpoint of any leaf of $\Lambda$.

Proof. Let $p, q$ be the fixed points of $g$. Suppose $p$ is an endpoint of a leaf $l$.

If the other endpoint of $l$ is not $q$, then the set $\left\{g^{\circ n}(l): n \in \mathbb{Z}\right\}$ gives an infinite set of leaves, each of which has $p$ as an endpoint, contradicting Lemma 4.3. Hence we may assume the other endpoint of $l$ is $q$.

Let $I$ be a connected component of $S^{1} \backslash\{p, q\}$. Define $\Lambda_{I}$ to be the set of leaves whose endpoints are in $I$ and visible from both $p$ and $q$. Assume $\Lambda_{I}$ is nonempty. Since $\{L\} \cup \Lambda_{I}$ bound a gap, say $P, \Lambda_{I}$ must be finite. But this means there must be a leaf in $\Lambda_{I}$ which connects $p$ to a point in $I$, which we already saw impossible. Now assume $\Lambda_{I}$ is empty. This means there exists a family of infinitely many leaves contained in $I$ which accumulate to $l$. But, since $g$ acts as a translation on $I$, this is impossible (if $l^{\prime}$ is a leaf close enough to $l$, then $l^{\prime}$ and $g l^{\prime}$ must be linked).

Theorem 4.5 (Solodov [23]). If $G$ is a subgroup of $\mathrm{Homeo}^{+}(\mathbb{R})$ such that each non-trivial element has at most one fixed point, and there is no global fixed point, then $[G, G]-\{I d\}$ consists of fixed-point-free elements.

Proof. See Step 4 in the proof of [23, Theorem 2.2.36]. 
Lemma 4.6. Let $\left(G, \Lambda_{1}, \Lambda_{2}\right)$ be a pseudo-fibered triple. Suppose $g \in G$ is properly pseudo-Anosov-like. If $h \in G$ shares a fixed point $p$ with $g$, then Fix $_{h}=$ Fix $_{g}$. In particular, $h$ is also a properly p-A-like element.

Proof. First we show that $h$ cannot be hyperbolic. Since $p$ is a fixed point of $g$, then, by Theorem 2.6, one of $\Lambda_{1}$ or $\Lambda_{2}$ contains a gap which has $p$ as a vertex. But then Lemma 4.4 says that $p$ cannot be a fixed point of a hyperbolic element. Therefore, by Theorem 2.6, $h$ must be pseudo-Anosov-like.

Without loss of generality, we may assume that $p$ is an attracting fixed point of both $g, h$, and $\Lambda_{1}$ contains the attracting polygon $P_{g}$ of $g$. Let $q$ be a vertex of $P_{g}$ which is connected to $p$ by a boundary leaf $l$ of $P_{g}$. If $q$ is not a fixed point of $h$, then $\left\{h^{\circ n}(l): n \in \mathbb{Z}\right\}$ is an infinite set of leaves which share $p$ as a common endpoint. This is impossible by Lemma 4.3 since $\Lambda_{1}$ is loose. This inductively shows that all vertices (i.e., all attracting fixed points of $g$ ) are fixed by $h$. Theorem 2.6 says there is no leaf connecting an attracting fixed point to a repelling fixed point for a given p-A-like element. Hence all attracting fixed points of elements of $g$ are attracting fixed points of $h$. Applying the same argument to the attracting polygon of $h$, one concludes that $P_{g}$ is in fact the attracting polygon of $h$ as well.

We showed the attracting polygon of $g$ and the attracting polygon of $h$ must coincide if $p$ is attracting. How about the repelling polygons? Note Theorem 2.6 (2) implies both the repelling polygon of $g$ and the repelling polygon of $h$ are contained in $\Lambda_{2}$.

Case 1. Suppose $g$ (hence also $h$ ) has at least three attracting fixed points. Since both $g$ and $h$ have a unique repelling fixed point between two adjacent vertices of $P_{g}$, the only way for the repelling polygons to be unlinked is that they coincide. Therefore, Fix $g=$ Fix $_{h}$.

Case 2. Now suppose $g$ has only two attracting fixed points. We know that each connected component of $S^{1} \backslash$ Fix $g$ has exactly one repelling fixed point of $g$ and exactly one repelling fixed point of $h$. Let $I$ be a connected component of $S^{1} \backslash$ Fix $g, r_{g}$ the repelling fixed point of $g$ on $I, r_{h}$ the repelling fixed point of $h$ on $I$. Let $H$ be the subgroup of $G$ generated by $g, h$. First note that every element of $H$ fixes the endpoints of $I$. From the previous arguments, we know that each element of $H$ is p-A-like and has exactly two attracting fixed points and two repelling fixed points. This implies that each element of $H$ has exactly one fixed point in $I$. Now we apply Theorem 4.5 by identifying $I$ with $\mathbb{R}$. If $r_{g} \neq r_{h}$, then $H$ has no global fixed point in $I$, and the theorem says that $H$ is abelian. But if $g$ and $h$ commute, then $r_{g}$ and $r_{h}$ coincide, a contradiction. Therefore, $r_{g}$ and $r_{h}$ must coincide from the beginning, and $\mathrm{Fix}_{g}=\mathrm{Fix}_{h}$ again. 
The following generalizes Lemma 4.6 for p-A-like elements that could be elliptic. We use $\operatorname{Per}_{f}$ to denote the set of all periodic points of a homeomorphism $f$.

Lemma 4.7. Let $g$ be a $p$-A-like element in a pseudo-fibered group $G$. If $h \in G$ shares a periodic point $p$ with $g$, then $\mathrm{Per}_{h}=\mathrm{Per}_{g}$, and $h$ is also $p$-A-like.

Proof. Take powers $g^{n}$ and $h^{m}$ such that $g^{n}$ is properly p-A-like and $p$ is fixed for $h^{m}$. Note that every periodic point of a properly p-A-like element is fixed. So Fix $g^{n}=\operatorname{Per}_{g}=\operatorname{Per}_{g}$. Applying Lemma 4.6, we get Fix $g^{n}=$ Fix $_{h^{m}}$ and that $h^{m}$ is properly p-A-like. So $h$ is p-A-like, and we also have $\operatorname{Per}_{h}=$ Fix $_{h^{m}}$, which agrees with Fix $_{g^{m}}=$ Per $_{g}$.

The previous lemma establishes "half" of the dynamical alternative. The other half follows from the next lemma.

Lemma 4.8. Let $G$ be a pseudo-fibered group. Suppose $g, h$ are hyperbolic elements of $G$ which share a fixed point $p$. Then every element of the subgroup generated by $g, h$ has the same fixed points as $g$.

Proof. Since $p$ is fixed by $g, h, g^{-1}, h^{-1}$, any element of the subgroup $H$ of $G$ generated by $g, h$ fixes $p$. But Lemma 4.6 says no p-A-like element shares a fixed point of a hyperbolic element. Hence all elements of such a subgroup must be hyperbolic.

Now we apply Theorem 4.5 to $H$ by identifying $S^{1} \backslash\{p\}$ with $\mathbb{R}$. Just as in Case 2 of the proof of Lemma 4.6, we conclude that $H$ has a (unique) global fixed point in $S^{1} \backslash\{p\}$.

Combining Lemmas 4.7 and 4.8 with Theorem 2.6, we immediately conclude Theorem B.

\subsection{Proof of Theorem A}

We now combine Theorem B with two known results to prove Theorem A. The first result is a very well-known tool in geometric group theory (for instance, see $[11$, Ch. II.B]).

Theorem 4.9 (Ping-pong lemma). Let $G$ be a group acting on a set $X$. Let $g_{1}, g_{2}$ be elements of $G$. Suppose there exist disjoint nonempty subsets $X_{1}^{+}, X_{1}^{-}, X_{2}^{+}, X_{2}^{-}$ of $X$ such that $g_{i}\left(X-X_{i}^{-}\right) \subset X_{i}^{+}, g_{i}^{-1}\left(X-X_{i}^{+}\right) \subset X_{i}^{-}$for each $i=1,2$. Then the subgroup generated by $g_{1}, g_{2}$ is free.

A proof of the second result we need can be found in many places, e.g., [17,23]. 
Theorem 4.10 (Hölder). Let $K$ be a subgroup of $\mathrm{Homeo}^{+}(\mathbb{R})$ which acts freely on $\mathbb{R}$. Then $K$ is abelian.

Now the ping-pong lemma implies the first case of the Tits alternative.

Lemma 4.11. Let $G$ be a pseudo-fibered group and $g_{1}, g_{2} \in G$. If $\operatorname{Per}_{g_{1}}$ and $\operatorname{Per}_{g_{2}}$ are disjoint, then there are powers of $g_{1}$ and $g_{2}$ that generate a non-abelian free subgroup of $G$.

Proof. We can replace $g_{1}$ and $g_{2}$ by some powers that satisfy $\operatorname{Per}_{g_{i}}=$ Fix $_{g_{i}}$. (If $g_{i}$ is hyperbolic, or properly p-A-like, no power needs to be taken. If $g_{i}$ is elliptic p-A-like, take a power that is properly p-A-like). Take $X_{i}^{+}$to be a neighborhood of the attracting fixed points of $g_{i}$ and $X_{i}^{-}$to be a neighborhood of the repelling fixed points of $g_{i}$ (for $i=1,2$ ). Since Fix $g_{1}$ and Fix $g_{2}$ are disjoint by hypothesis, we can take $X_{i}^{+}, X_{i}^{-}$(for $i=1,2$ ) to be all disjoint. Now let $h_{i}$ be a high enough power of $g_{i}$ so that $h_{i}\left(S^{1}-X_{i}^{-}\right) \subset X_{i}^{+}$and $h_{i}^{-1}\left(X-X_{i}^{+}\right) \subset X_{i}^{-}$for $i=1,2$. This is possible because of the dynamics of hyperbolic and properly p-A-like elements. By the ping-pong lemma, $h_{1}$ and $h_{2}$ generate a free subgroup.

Hölder's theorem implies the alternative case.

Lemma 4.12. Let $G$ be a pseudo-fibered group, $g \in G$ and $P=\operatorname{Per}_{g}$. Consider the subgroup $H=\{h \in G: h P=P\}$ of $G$ that leaves $P$ invariant. Then

(1) $H=\left\{h \in G: \operatorname{Per}_{h}=P\right\}$,

(2) $H$ is virtually abelian.

Proof. Let $h \in H$. Since $h P=P$ and $P=\operatorname{Per}_{g}$ is finite, then $P \subset \operatorname{Per}_{h}$. Then, by Theorem B, we get $\operatorname{Per}_{h}=\operatorname{Per}_{g}=P$. This proves the first assertion, the other inclusion being trivial.

For the second assertion, let $K \unlhd H$ consist of elements that stabilize $\operatorname{Per}_{g}$ pointwise. That is, $K=\left\{h \in H \mid \mathrm{Fix}_{h}=\operatorname{Per}_{g}\right\}$. Note

$$
[H: K] \leq \frac{\left|\operatorname{Per}_{g}\right|}{2},
$$

so, in particular, to show $H$ is virtually abelian, it suffices to show $K$ is abelian. Assertion (1) implies $K$ acts freely on each component of $S^{1} \backslash \operatorname{Per}_{g}$, each of which is an interval. So, by Hölder's theorem, $K$ is abelian.

Theorem A now follows from Theorem B since, if $H$ is a subgroup of $G$, then either there exist two elements of $H$ with distinct periodic point sets or $H$ has a global set of periodic points. In the first case, apply Lemma 4.11; in the second case, apply Lemma 4.12. 


\subsection{Remarks on the proofs of Theorems A and B}

Conjecturally, non-elementary pseudo-fibered groups are word-hyperbolic. For word-hyperbolic groups, a stronger version of the Tits alternative holds: an infinite subgroup of a word-hyperbolic group either contains a free group of rank 2 or is virtually cyclic. To obtain this stronger Tits alternative, one needs to strengthen Lemma 4.12. Let $H$ be a subgroup as in Lemma 4.12, and assume it is actually abelian. Then Ghys [17] provides an $H$-invariant measure on each connected component of $S^{1}-\operatorname{Per}_{H}$. The problem is that this measure may not have full support. Even when the action of $G$ on $S^{1}$ is minimal, it is still not clear if it can be shown that we have an invariant measure of full support on the complement of $\operatorname{Per}_{H}$. The stronger Tits alternative would easily follow from there.

More directly, it is easy to verify that this stronger Tits alternative is equivalent to showing that the subgroup $K$ in the proof of Lemma 4.12 is isomorphic to $\mathbb{Z}$. A stronger version of Hölder's theorem says that, for any abelian subgroup of $\mathrm{Homeo}^{+}(\mathbb{R})$, there is a blow-down of $\mathbb{R}$ such that the induced action of the subgroup is faithful and by translations. Thus showing $K$ is $\mathbb{Z}$ is equivalent to showing that this translation action is not minimal.

Finally, we remark that, if we knew that pseudo-Anosov-like elements really were pseudo-Anosov, the stronger Tits alternative would follow from the fact that two pseudo-Anosovs commute if and only if they are powers of some other pseudo-Anosov. This can be seen by considering the action of the mapping class group on Thurston's compactification of Teichmüller space: Two pseudo-Anosovs must fix the same axis if they commute. The mapping class group acts discretely on Teichmüller space; hence the subgroup generated by the two pseudo-Anosovs must act discretely on the axis in Teichmüller space. Each pseudo-Anosov acts by translations on this axis, so we conclude that the subgroup the two generate is isomorphic to $\mathbb{Z}$.

\section{Purely hyperbolic pseudo-fibered groups and convergence group actions on $S^{2}$}

We now restrict our attention to the special class of purely hyperbolic pseudofibered groups. In [1], it was conjectured that such groups are always Fuchsian or, equivalently, convergence subgroups of $\mathrm{Homeo}^{+}\left(S^{1}\right)$. While this conjecture remains open, Theorem $C$ shows that, as expected, purely hyperbolic pseudo-fibered groups act on the 2-sphere as convergence groups. We remark that a similar idea of relating group action on $S^{1}$ with a geometric origin to convergence group action on $S^{2}$ has been carried out in the context of pseudo-Anosov flows in Fenley's program [12, Section 4]. 
To prove Theorem $\mathrm{C}$, we begin by reviewing the construction of [1], which is responsible for the existence of an $S^{2}$ on which a pseudo-fibered group can act, and inspired by results of Cannon and Thurston [8]. Moore's theorem [22] implies that, for any pseudo-fibered triple $\left(G, \Lambda_{1}, \Lambda_{2}\right)$, there exists a quotient map $\pi: S^{1} \rightarrow S^{2}$, constructed by first identifying two disks laminated by $\Lambda_{1}$ and $\Lambda_{2}$ (respectively) along their common boundary $S^{1}$ and then collapsing all the gaps of the $\Lambda_{i}$ to points. Since each lamination is $G$-invariant, this induces a $G$-action on $S^{2}$ such that $\pi$ is $G$-equivariant. We call this map $\pi$ the Cannon-Thurston map for the pseudo-fibered triple $\left(G, \Lambda_{1}, \Lambda_{2}\right)$. For details, one can also consult [ 8 , Section 14]. A basic observation about this construction is the following lemma.

Lemma 5.1. Let $\left(G, \Lambda_{1}, \Lambda_{2}\right)$ be a pseudo-fibered triple. Suppose there exists a sequence $\left(\bar{x}_{i}\right)$ of points in $S^{2}\left(=\pi\left(S^{1}\right)\right)$ which converges to $\bar{x}$, and a sequence $\left(g_{i}\right)$ of elements of $G$ such that $g_{i}\left(\bar{x}_{i}\right)$ converges to $\bar{x}^{\prime}$ in $S^{2}$. Then, passing to subsequences if necessary, there exists a sequence $\left(x_{i}\right)$ of points in $S^{1}$ converging to $x$ such that $g_{i}\left(x_{i}\right)$ converges to $x^{\prime}$ in $S^{1}$, where $\bar{x}_{i}=\pi\left(x_{i}\right)$ and $\bar{x}^{\prime}=\pi\left(x^{\prime}\right)$.

Proof. This is straightforward because $S^{1}$ is compact, and $\pi$ is continuous, surjective and $G$-equivariant.

We now state a few dynamical lemmas, after which we will prove Theorem C.

Lemma 5.2. Let $G$ be a group acting on $S^{1}$ such that there exists a $G$-invariant lamination with a rainbow at $p \in S^{1}$. Suppose there exists a sequence $\left(g_{i}\right)$ of elements of $G$ such that, for any neighborhood $U$ of $p, g_{i}(U)$ intersects $U$ nontrivially for all large $i$. Then $p$ is an accumulation point of some fixed points of the elements in the sequence $\left(g_{i}\right)$.

Proof. See the proof of [1, Proposition 7.5].

Lemma 5.3. Let $\left(G, \Lambda_{1}, \Lambda_{2}\right)$ be a pseudo-fibered triple. Suppose $\left(x_{i}\right)$ is a sequence of points in $S^{1}$ which converges to $x$, and there exists a sequence $\left(g_{i}\right)$ of elements of $G$ such that $g_{i}\left(x_{i}\right)$ converges to $x^{\prime}$. Then either $x$ is an accumulation points of fixed points of the sequence $\left(g_{i+1}^{-1} \circ g_{i}\right)$ or $x^{\prime}$ is an accumulation point of fixed points of the sequence $\left(g_{i+1} \circ g_{i}^{-1}\right)$. Moreover, if $x_{i}=x$ for all $i$, then $x^{\prime}$ must be an accumulation point of fixed points of (any subsequence of) the sequence $\left(g_{i+1} \circ g_{i}^{-1}\right)$.

Proof. This is a straightforward consequence of Lemma 5.2. See, for instance, the proof of [1, Proposition 7.6].

Proof of Theorem C. Suppose the negation of the conclusion. Then there exists an infinite sequence of distinct elements $\left(g_{i}\right)$ of $G$ which does not have the conver- 
gence property, i.e., the set $\left\{g_{i}\right\}$ does not act properly discontinuously on the set of triples of distinct points of $S^{2}$. More precisely, this means that, after passing to a subsequence of $\left(g_{i}\right)$, there exist three convergent sequences in $S^{2}$,

$$
\bar{x}_{i} \rightarrow \bar{x}, \quad \bar{y}_{i} \rightarrow \bar{y}, \quad \bar{z}_{i} \rightarrow \bar{z}, \quad \bar{x}_{i} \neq \bar{y}_{i} \neq \bar{z}_{i} \neq \bar{x}_{i}, \quad \bar{x} \neq \bar{y} \neq \bar{z} \neq \bar{x},
$$

and three elements $\bar{x}^{\prime}, \bar{y}^{\prime}, \bar{z}^{\prime} \in S^{2}$ such that

$$
g_{i}\left(\bar{x}_{i}\right) \rightarrow \bar{x}^{\prime}, \quad g_{i}\left(\bar{y}_{i}\right) \rightarrow \bar{y}^{\prime}, \quad g_{i}\left(\bar{z}_{i}\right) \rightarrow \bar{z}^{\prime}, \quad \bar{x}^{\prime} \neq \bar{y}^{\prime} \neq \bar{z}^{\prime} \neq \bar{x}^{\prime} .
$$

We conclude that there exist sequences and points in $S^{1}$ such that

$$
x_{i} \rightarrow x, \quad y_{i} \rightarrow y, \quad z_{i} \rightarrow z, \quad x_{i} \neq y_{i} \neq z_{i} \neq x_{i}, \quad x \neq y \neq z \neq x
$$

and three elements $x^{\prime}, y^{\prime}, z^{\prime} \in S^{1}$ such that

$$
g_{i}\left(x_{i}\right) \rightarrow x^{\prime}, \quad g_{i}\left(y_{i}\right) \rightarrow y^{\prime}, \quad g_{i}\left(z_{i}\right) \rightarrow z^{\prime}, \quad x^{\prime} \neq y^{\prime} \neq z^{\prime} \neq x^{\prime},
$$

where, in our notation, $p$ is some fixed point in the preimage of $\bar{p}$ under $\pi$ for any $\bar{p} \in S^{2}$, in accordance with Lemma 5.1. In words, we can lift sequences exhibiting the failure of $G$ to act as a convergence group on $S^{2}$ to sequences exhibiting the failure of $G$ to act as a convergence group on $S^{1}$.

Since $\Lambda_{1}$ and $\Lambda_{2}$ do not share any endpoints, for each $p \in\{x, y, z\}$, there exists a rainbow at $p$ in at least one of the $\Lambda_{i}$. In particular, for each $p \in\{x, y, z\}$, there exists a leaf $L_{p}$ which separates $p$ from the other two points in $\{x, y, z\} \backslash\{p\}$ (which lamination $L_{p}$ belongs to is not important, and $L_{x}, L_{y}, L_{z}$ are not necessarily leaves of the same lamination). Passing to a subsequence, we may assume that each of the sequences of pairs of points described by the endpoints of the leaves $\left(g_{i}\left(L_{x}\right)\right),\left(g_{i}\left(L_{y}\right)\right),\left(g_{i}\left(L_{z}\right)\right),\left(g_{i}^{-1}\left(L_{x}\right)\right),\left(g_{i}^{-1}\left(L_{y}\right)\right),\left(g_{i}^{-1}\left(L_{z}\right)\right)$ converges to a pair of points, which are possibly not distinct.

By Lemma 5.3, either at least two of $x, y$ and $z$ are accumulation points of fixed points of the sequence $\left(g_{i+1}^{-1} \circ g_{i}\right)$ or at least two of $x^{\prime}, y^{\prime}$ and $z^{\prime}$ are accumulation points of fixed points of the sequence $\left(g_{i+1} \circ g_{i}^{-1}\right)$. Without loss of generality, we assume that $x^{\prime}$ and $y^{\prime}$ are accumulation points of fixed points of the sequence $\left(g_{i+1} \circ g_{i}^{-1}\right)$, possibly after exchanging the roles of $g_{i}$ and $g_{i}^{-1}$. Furthermore, since each element of $G$ has exactly two fixed points, there exists a subsequence $\left(g_{i_{j}+1} \circ g_{i_{j}}^{-1}\right)$ of the sequence $\left(g_{i+1} \circ g_{i}^{-1}\right)$ such that $x^{\prime}$ and $y^{\prime}$ are the only accumulation points of the fixed points of the $g_{i_{j}+1} \circ g_{i_{j}}^{-1}$. By the second statement of Lemma 5.3, if $p \in S^{1}$ is such that $g_{i}(p)$ converges to $p^{\prime}$, then $p^{\prime}$ must be either $x^{\prime}$ or $y^{\prime}$. In particular, considering the sequence $\left(g_{i}\left(L_{z}\right)\right)$, which converges to some pair of possibly non-distinct points $\left\{e_{1}, e_{2}\right\}$, what we have shown implies each $e_{i}$ is either $x^{\prime}$ or $y^{\prime}$.

If $e_{1} \neq e_{2}$, since laminations are required to be closed, the limit of the sequence of leaves $\left.\left(g_{i}\left(L_{z}\right)\right)\right)$ must be a leaf connecting $x^{\prime}$ to $y^{\prime}$ in the lamination $\Lambda_{i}$ contain- 
ing $L_{z}$. But, since $\bar{x}^{\prime}=\pi\left(x^{\prime}\right)$ and $\bar{y}^{\prime}=\pi\left(y^{\prime}\right)$ are assumed to be distinct, by the definition of the Cannon-Thurston map $\pi$, their preimages cannot be connected by a leaf. This contradiction implies $e_{1}=e_{2}$.

Let us assume that $e_{1}=e_{2}=x^{\prime}$. We will show that $x^{\prime}$ is not distinct from both $y^{\prime}$ and $z^{\prime}$. In the case $e_{1}=e_{2}=y^{\prime}$, the same argument would lead us to contradict that $y^{\prime}$ is not distinct from both $x^{\prime}$ and $z^{\prime}$.

Let $I_{y}$ be the closure of the connected component of $S^{1}-L_{z}$ which contains $y$, and define $I_{z}$ similarly for $z$. Take a nested sequence of closed neighborhoods $\left(U_{i}\right)$ of $x^{\prime}$ such that $U_{i} \rightarrow x^{\prime}$ as $i \rightarrow \infty$. Passing to a subsequence, one may assume that the endpoints of $g_{i}\left(L_{z}\right)$ are contained in $U_{i}$.

For each $i$, there are two possibilities: either $g_{i}\left(I_{z}\right) \subset U_{i}$ or $g_{i}\left(I_{y}\right) \subset U_{i}$. Suppose the former happens for infinitely many $i$. Since $\left(z_{i}\right)$ converges to $z$, then, for all large enough $i, z_{i}$ is in $I_{z}$. Hence $g_{i}\left(z_{i}\right) \in U_{i}$ for infinitely many $i$, so some subsequence of $g_{i}\left(z_{i}\right)$ converges to $x^{\prime}$. But this is impossible since $z^{\prime}$ is assumed to be distinct from $x^{\prime}$, and $g_{i}\left(z_{i}\right)$ converges to $z^{\prime}$. If instead $g_{i}\left(I_{y}\right) \subset U_{i}$ for infinitely many $i$, then we similarly contradict the assumption that $y^{\prime} \neq x^{\prime}$.

We remark that this proof almost goes through to show that a purely hyperbolic pseudo-fibered group $G$ acts as a convergence group on the circle $S^{1}=\pi^{-1}\left(S^{2}\right)$. The only gap to consider is the case where $e_{1} \neq e_{2}$.

We conclude the paper with some questions which naturally arise from the results of this paper.

Question 5.4. For fibered groups, how do hyperbolic elements and p-A-like elements interact? What can be said about the group structure of a pseudo-fibered group in terms of the dynamical feature of the elements of the group?

Question 5.5. For a fibered group action on $S^{1}$, without using hyperbolic geometry, can we abstractly show that the induced action on $S^{2}$ as in this section is a uniform convergence group?

Answering (or rather understanding) above questions appropriately, one might hope to get a characterization of fibered hyperbolic 3-manifold groups via its action on $S^{1}$ with invariant laminations. For instance, assuming Cannon's conjecture [7], an affirmative answer to Question 5.5 together with a result of Bowditch [3] would imply that such a group is always a closed hyperbolic 3-manifold group.

Acknowledgments. We thank David Cohen for asking the second author if the Tits alternative holds for pseudo-fibered groups. We also wish to thank Danny Calegari, Ursula Hamenstädt and Dawid Kielak for helpful conversations. We greatly appreciate for the anonymous referee for the valuable comments which 
improved the structure of the paper significantly. The third author thanks Universität Bonn for hospitality, during which time some of this work was completed.

\section{Bibliography}

[1] H. Baik, Fuchsian groups, circularly ordered groups and dense invariant laminations on the circle, Geom. Topol. 19 (2015), no. 4, 2081-2115.

[2] H. Baik and E. Samperton, Spaces of invariant circular orders of groups, Groups Geom. Dyn. 12 (2018), no. 2, 721-763.

[3] B. H. Bowditch, A topological characterisation of hyperbolic groups, J. Amer. Math. Soc. 11 (1998), no. 3, 643-667.

[4] D. Calegari, Foliations and geometrization of 3-manifolds, Lecture note for the course 'Foliations and 3-manifolds' at University of Chicago, (2003).

[5] D. Calegari, Foliations and the Geometry of 3-manifolds, Oxford Math. Monogr., Oxford University Press, Oxford, 2007.

[6] D. Calegari and N. M. Dunfield, Laminations and groups of homeomorphisms of the circle, Invent. Math. 152 (2003), no. 1, 149-204.

[7] J. W. Cannon and E. L. Swenson, Recognizing constant curvature discrete groups in dimension 3, Trans. Amer. Math. Soc. 350 (1998), no. 2, 809-849.

[8] J. W. Cannon and W.P. Thurston, Group invariant Peano curves, Geom. Topol. 11 (2007), 1315-1355.

[9] A. Casson and D. Jungreis, Convergence groups and Seifert fibered 3-manifolds, Invent. Math. 118 (1994), no. 3, 441-456.

[10] A. J. Casson and S. A. Bleiler, Automorphisms of Surfaces after Nielsen and Thurston, London Math. Soc. Stud. Texts 9, Cambridge University Press, Cambridge, 1988.

[11] P. de la Harpe, Topics in Geometric Group Theory, Chicago Lectures in Math., University of Chicago Press, Chicago, 2000.

[12] S. Fenley, Ideal boundaries of pseudo-Anosov flows and uniform convergence groups with connections and applications to large scale geometry, Geom. Topol. 16 (2012), no. 1, 1-110.

[13] S. Fenley and T. Barbot, Free seifert pieces of pseudo-anosov flows, preprint (2015), https://arxiv.org/abs/1512.06341.

[14] S. R. Fenley, Quasigeodesic pseudo-Anosov flows in hyperbolic 3-manifolds and connections with large scale geometry, Adv. Math. 303 (2016), 192-278.

[15] S. Frankel, Quasigeodesic flows and sphere-filling curves, Geom. Topol. 19 (2015), no. $3,1249-1262$. 
[16] D. Gabai, Convergence groups are Fuchsian groups, Bull. Amer. Math. Soc. (N. S.) 25 (1991), no. 2, 395-402.

[17] E. Ghys, Groups acting on the circle, Enseign. Math. (2) 47 (2001), no. 3-4, 329-407.

[18] A. Hinkkanen, Abelian and nondiscrete convergence groups on the circle, Trans. Amer. Math. Soc. 318 (1990), no. 1, 87-121.

[19] N. Kovačević, Examples of Möbius-like groups which are not Möbius groups, Trans. Amer. Math. Soc. 351 (1999), no. 12, 4823-4835.

[20] K. Mann, Spaces of surface group representations, Invent. Math. 201 (2015), no. 2, $669-710$.

[21] G. Margulis, Free subgroups of the homeomorphism group of the circle, C. R. Acad. Sci. Paris Sér. I Math. 331 (2000), no. 9, 669-674.

[22] R.L. Moore, Concerning upper semi-continuous collections of continua, Trans. Amer. Math. Soc. 27 (1925), no. 4, 416-428.

[23] A. Navas, Groups of Circle Diffeomorphisms, Chicago Lectures in Math., University of Chicago Press, Chicago, 2011.

[24] E. Te Winkel, Sticky and slippery laminations on the circle, Master's thesis, University of Bonn, 2016.

[25] W. P. Thurston, Three-manifolds, foliations and fircles, I, preprint (1997), https : //arxiv.org/abs/math/9712268v1.

[26] P. Tukia, Homeomorphic conjugates of Fuchsian groups, J. Reine Angew. Math. 391 (1988), 1-54.

[27] P. Tukia, Convergence groups and Gromov's metric hyperbolic spaces, New Zealand J. Math. 23 (1994), no. 2, 157-187.

Received October 10, 2017; revised January 16, 2019.

\section{Author information}

Juan Alonso, Centro de Matemática, Facultad de Ciencias,

Universidad de la República, Iguá 4225, Montevideo C.P. 11400, Uruguay.

E-mail: juan@cmat.edu.uy

Hyungryul Baik, Department of Mathematical Sciences, KAIST,

291 Daehak-ro Yuseong-gu, Daejeon, 34141, South Korea.

E-mail: hrbaik@kaist.ac.kr

Eric Samperton, Department of Mathematics, South Hall,

Room 6607, University of California, Santa Barbara, CA 93106-3080, USA.

E-mail: eric@math.ucsb.edu 